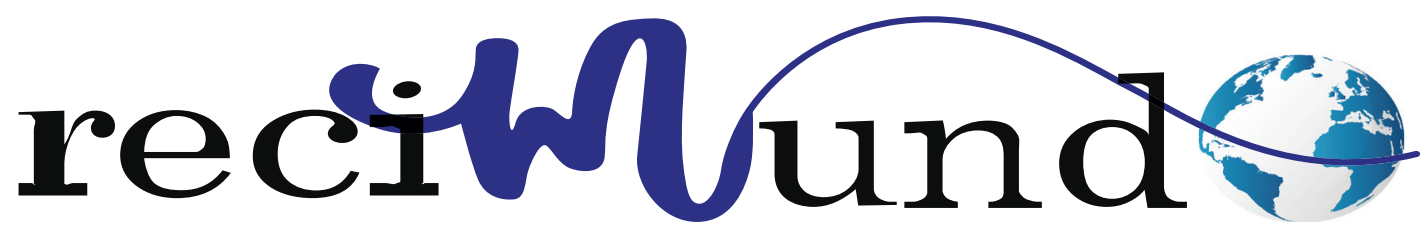

Revista Científica Mundo de la Investigación y el Conocimiento

DOI: 10.26820/recimundo/5.(3).sep.2021.359-367

URL: https://recimundo.com/index.php/es/article/view/1283

EDITORIAL: Saberes del Conocimiento

REVISTA: RECIMUNDO

ISSN: 2588-073X

TIPO DE INVESTIGACIÓN: Artículo de revisión

CÓDIGO UNESCO: 3213.13 Estomatología

PAGINAS: $359-367$

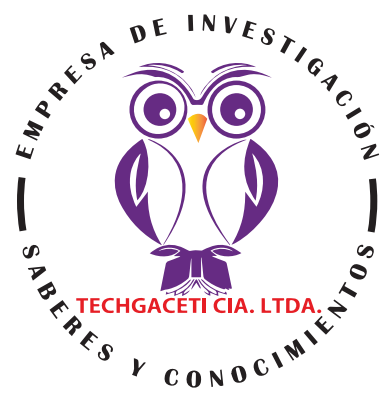

Enfermedad periodontal, prevalencia y factores de riesgo en niños y adolescentes. Revisión de la literatura

Digitally guided surgery: advances and perspectives on 3D digital technology in periodology and implantology

Cirurgia Digitalmente Guiada: Avanços e perspectivas da tecnologia digital 3D em Periodontia e Implantologia

Andrés Josue Franco Mejía'; Mariela Cumanda Balseca Ibarra²

RECIBIDO: 20/06/2021 ACEPTADO: 15/07/2021 PUBLICADO: 20/07/2021

1. Egresado del Posgrado de Periodoncia; Facultad de Odontología de la Universidad Central del Ecuador; Odontólogo; Quito, Ecuador; ajfranco@uce.edu.ec; https://orcid.org/0000-0003-2099-2644

2. Doctora en Ciencias en el Programa: Ciencias Odontológicas, Área de concentración: Periodoncia; Especialista en Periodoncia; Doctora en Odontología; Docente de la Facultad de Odontología de la Universidad Central del Ecuador; Quito, Ecuador; mbalseca@uce.edu.ec; https://orcid.org/0000-0002-7218-3622

\title{
CORRESPONDENCIA
}

Andrés Josue Franco Mejía

ajfranco@uce.edu.ec

\section{Quito, Ecuador}

() RECIMUNDO; Editorial Saberes del Conocimiento, 2021 


\section{RESUMEN}

Las enfermedades periodontales son una de las principales causas de morbilidad en la persona adulta, siendo esta la principal causa de pérdida de órganos dentarios junto con la caries. Sin embargo, y a pesar de la vasta literatura sobre esta patología, se ha descrito poca información de las periodontopatías en los niños. Aspectos biológicos, inmunológicos, microbiológicos e incluso metodológicos han impedido llevar a cabo proyectos relacionados con enfermedad periodontal en individuos menores de 12 años, motivo por el cual el presente estudio se enfocará en describir mediante la recopilación de datos en inglés, español y portugués de diversas bases científicas el estado periodontal de este grupo poblacional en particular.

Palabras clave: Enfermedad periodontal, prevalencia, factores de riesgo, niños.

\section{ABSTRACT}

Periodontal diseases are one of the main causes of morbidity in adults, this being the main cause of loss of dental organs along with caries. However, and despite the vast literature on this pathology, little information on periodontal disease in children has been described Biological, immunological, microbiological and even methodological aspects have prevented carrying out projects related to periodontal disease in individuals under 12 years of age, which is why this study will focus on describing by collecting data in English, Spanish and Portuguese of various scientific bases the periodontal status of this population group in particular.

Keywords: Periodontal disease, prevalence, risk factors, children.

\section{RESUMO}

As doenças periodontais são uma das principais causas de morbidade em adultos, sendo esta a principal causa de perda de órgãos dentais juntamente com a cárie. No entanto, e apesar da vasta literatura sobre essa patologia, poucas informações sobre a doença periodontal em crianças foram descritas. Aspectos biológicos, imunológicos, microbiológicos e até metodológicos têm impedido a realização de projetos relacionados à doença periodontal em menores de 12 anos, razão pela qual este estudo se concentrará em descrever por meio de coleta de dados em inglês, espanhol e português de diversas bases científicas a doença periodontal status desse grupo populacional em particular.

Palavras-chave: Doença periodontal, prevalência, fatores de risco, crianças. 


\section{Introducción}

En la actualidad, es internacionalmente aceptado que la segunda causa de morbilidad bucal es la inflamación gingival, empezando desde sus fases iniciales como gingivitis, hasta evolucionar a periodontitis, que puede conllevar a la pérdida de los órganos dentarios afectados ${ }^{1}$.

Algunos estudios refieren que la gingivitis afecta a más del 90 por ciento de la población. Esta alta probabilidad de adquirir fácilmente la enfermedad obedecería a una higiene oral deficiente, lo que permite el acúmulo de biopelícula y desarrollar con rapidez estados inflamatorios en la encía².

Sin embargo, esta patología muy recurrente en el adulto ha sido poco descrita en los niños, a pesar de que estadios iniciales de las periodontopatías se pueden evidenciar incluso antes de la presencia de dientes permanentes. Claramente la gingivitis es la enfermedad periodontal más común en este grupo etario, no obstante, condiciones adicionales como la periodontitis han sido poco descritas, especialmente por factores biológicos, metodológicos y microbiológi$\cos ^{3-5}$.

El presente artículo describirá mediante una revisión y análisis de la literatura, la prevalencia de enfermedad periodontal, así como los principales factores de riesgo asociados, en niños y adolescentes. A pesar de que la información que se va a encontrar sobre este tema será limitada, se espera encontrar que la gingivitis sea la enfermedad periodontal más común en niños y adolescentes, siendo esta causada por el estímulo bacteriano, y agravada por factores de riesgo locales tales como restauraciones desbordantes, cálculos, apiñamiento dental entre otros; y sistémicos como diabetes, síndrome de Down, y obesidad. De igual manera, se espera que la periodontitis sea una enfermedad poco frecuente en este grupo etario, pudiendo estar presente en grupos específicos que tengan factores sistémicos agravantes como las enfermedades descritas anteriormente.

\section{Metodología}

El presente artículo es una revisión de la literatura, que recopila y sintetiza información de artículos científicos (revisiones sistemáticas, casos clínicos, revisiones de la literatura, etc.) de los últimos 20 años sobre la enfermedad periodontal en niños y adolescentes. Se utilizarán como referencia palabras claves como "gingivitis", "periodontitis", "factores de riesgo" y se aplicarán filtros por edades y género. Se incluirán artículos en idioma inglés, español y portugués.

\section{Resultados}

\section{Prevalencia}

La enfermedad periodontal con mayor prevalencia es la gingivitis, mostrando altos índices en Latinoamérica en niños y adolescentes, según autores como Botero, Orozco, Balseca. Botero en su revisión sistemática recalca que esta patología afecta al 34,7 por ciento en promedio de la población Latinoamericanaica infantil y adolescente, encontrando una alta prevalencia del 77 por ciento y del 73 por ciento en países como Colombia y Bolivia respectivamente ${ }^{1,2,6}$.

Mientras que, en estudios llevados a cabo en Brasil también denotan una alta tasa de gingivitis en adolescentes de 12 años. Biazevick en el 2008 encontró en el mismo país índices de sangrado relativamente menores, que oscilaban aproximadamente entre el 39, 68 por ciento?.

En tanto, en países como Colombia, Orozco menciona la prevalencia de gingivitis cercana al 18 por ciento. Además, se encontró que de las 116 personas examinadas de doce años en adelante, el 11 por ciento tenía bolsas de $5.5 \mathrm{~mm}$ o más ${ }^{6}$. 
Chile también presenta estudios relevantes en cuanto al tema de salud periodontal. Soto 2007 en un estudió que involucró a 2232 adolescentes de 12 años, obtuvo una prevalencia del 67 por ciento. Además aclaró que la población rural se veía más afectada por esta patología que la población urbana ${ }^{8}$.

En 1996 en Ecuador se realizó un levantamiento epidemiológico por parte del Ministerio de Salud pública del Ecuador, en donde se evaluaron patologías como caries dental, periodontopatías y fluorosis. El grupo de estudio consistió en niños y adolescentes entre 12 a 15 años, con una población total de 2600 individuos donde se demostró que el 65,6 por ciento de la población presentaba algún tipo de alteración periodontal, que incluía cierto tipo de inflamación, sangrado o edema de la encía? .

Finalmente, en un estudio más reciente realizado por Balseca en 998 escolares de la ciudad de Quito, encontró que el 93 por ciento de los examinados tenían sangrado gingival. Además el estudio concluyó que el sangrado gingival afectaba de manera negativa la calidad de vida de los individuos ${ }^{2}$.

\section{Factores de Riesgo: Higiene Oral}

La biopelícula es el factor etiológico primario de la enfermedad gingival; sin embargo, este puede ser controlado mecánicamente y con procedimientos complementarios (seda dental, cepillos interdentales, etc.) Un ejemplo de la importancia del autocontrol minucioso de la biopelicula es que Socransky, citado en Lindhe, confirmó que: “... un régimen de control de la placa supragingival óptimo permanente puede modificar la composición de la micro flora de la bolsa y reducir el porcentaje de bacterias" ${ }^{10}$.

En la actual clasificación de la enfermedad periodontal (2017) se ha redefinido a los factores de riesgo como factores predisponentes y factores modificantes. Un factor predisponente se define como cualquier elemento que provoque mayor acumulación de biopelícula, y con ello una mayor probabilidad de que desarrolle enfermedad periodontal. Por otra parte, un factor modificante se puede definir como cualquier componente que modifique la respuesta del huésped ante la biopelícula, siendo el principal ejemplo las enfermedades sistémicas ${ }^{11}$.

La realidad es que a pesar de que muchos pacientes dicen cepillarse sus dientes al menos dos veces por día, estudios epidemiológicos y clínicos demuestran que los procedimientos mecánicos de la mayoría de la población son insuficientes para controlar la biopelícula,, permitiéndonos concluir que existe una deficiencia en la duración y/o realización de la técnica, acompañado por la falta de uso de agentes interproximales de limpieza como por ejemplo el hilo dental y cepillos dentales interproximales ${ }^{12}$.

En un estudio realizado por Juárez en 2004 sobre la eficacia de higiene oral, evaluando a un grupo de 382 niños entre 4 y 6 años, mediante el índice de higiene oral simplificado, donde se demostró una inadecuada higiene oral, que estaba directamente relacionada con la gingivitis o cuadros más severos de esta. De un total de 149 niños con higiene oral deficiente, 116 presentaron algún tipo de cuadro inflamatorio $1^{3}$.

De igual manera otro estudio realizado en México demostró el impacto de una mala higiene oral en el desarrollo de periodontopatías. Uno de los estudios en un total de 1527 pacientes, estableció que el parámetro principal fue la cantidad de biopelícula en la superficie dental, encontrándose una asociación positiva, y estadísticamente significativa entre la biopelícula, y la gingivitis, obteniéndose una razón de riesgo de 2,44 mayor que los pacientes con ausencia de biopelícula, visible ${ }^{14}$. 


\section{Cálculo dental}

En cuanto a la presencia de cálculo como factor de riesgo para el aparecimiento de la enfermedad periodontal es un tema controversial, pues el cálculo está siempre cubierto por una capa de biofilm no mineralizado, lo que dificulta determinar si el cálculo como tal, tiene un efecto perjudicial sobre el tejido periodontal per se, o es la biopelícula vital el causante de la enfermedad ${ }^{15-16}$.

A pesar de las fuertes asociaciones entre el cálculo y periodontitis en estudios experimentales y epidemiológicos, se debe tener en cuenta que el cálculo en las periodontopatías parece jugar un papel nocivo desde el punto de vista retentivo para la biopelícula más que el de un agente etiológico como tal. Grandes cantidades de cálculo obstruyen la eficacia de la higiene bucal diaria y, por consiguiente, aceleran la formación de biopelícula, pudiendo ser esta la verdadera etiología de la enfermedad ${ }^{15}$.

Juárez en este mismo estudio, observó que los pacientes que presentaban cálculo tenían mayor incidencia de cuadros inflamatorios gingivales, específicamente de los 20 niños de entre 4 y 6 años, 14 presentaron un cuadro inflamatorio gingival, no obstante en el presente estudio esta diferencia no fue

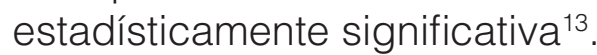

\section{Síndrome de Down (DS) y enfermedad periodontal en niños}

La enfermedad periodontal en pacientes con síndrome de Down es grave, generalizada, de rápida progresión y clasificada como manifestación de enfermedades sistémicas asociadas a trastornos genéticos por Academia Americana de Periodoncia ${ }^{17}$.

Johnson y Young citado por Morgan 2017 examinaron a 70 niños con síndrome de Down en una edad media de 3 a 10 años y los comparó con 40 personas que no tenían este síndrome, pero que tenían discapaci- dades de aprendizaje similares, observando que el $96 \%$ de las personas en el grupo con síndrome de Down presentaron enfermedad periodontal ${ }^{18}$.

El aumento de la prevalencia y la gravedad enfermedad periodontal en personas con síndrome de Down puede atribuirse a factores locales asociados con la cavidad oral, así como factores sistémicos asociado con el trastorno genético en sí18. $^{18}$.

La enfermedad periodontal en personas con síndrome de Down, puede comenzar entre los 6 y los 15 años de edad, esto puede deberse a que su capacidad para mantener un adecuado control de biopelícula es reducida, y esto a su vez podría estar asociado a un deterioro de la motricidad fina, característico de este síndrome, función que resulta en una destreza manual deficiente ${ }^{19}$.

Swallow citado por Morgan 2017 informó que el 82,9 por ciento de los niños con DS tenían los labios habitualmente separados, lo que conduce a una respiración bucal, por lo que existe una sequedad de la misma y por ello menos salivación, a su vez Morgan apoya la teoría de que estos pacientes presentan una inmunidad deteriorada debido a una reducción en la quimiotaxis de neutrófilos, fagocitosis de neutrófilos y función de los linfocitos T, así como aumento de la producción de mediadores de la inflamación y enzimas proteolíticas ${ }^{17}$.

\section{Diabetes}

La diabetes se caracteriza por ser una enfermedad crónica que repercute negativamente en la salud periodontal, siendo la periodontitis una de las complicaciones bucales más frecuentes en el paciente diabético. No obstante, esta asociación es menos sencilla de explicar en pacientes jóvenes o niños. Un estudio realizado por Manoucher concluyó que la enfermedad periodontal inicia en la pubertad y progresa rápidamente a lo largo de los años de manera más

\section{2.}


rápida en el paciente diabético. De igual manera Lalla y colaboradores demostraron que la destrucción periodontal puede iniciar incluso a edades tempranas y que puede hacerse más evidente en el crecimiento de niño a adolescente ${ }^{20,21}$.

Un estudio más específico de personas entre 6 y 18 años, llevado a cabo en 2008 comparó 187 individuos con diabetes tipo I, y 178 sin diabetes, pudiendo concluir que el grupo con diabetes tenía mayor inflamación gingival, y destrucción periodontal, que el grupo control. Además, la razón de riesgo (OD) de que la diabetes sea un factor de riesgo principal para la periodontitis fue de $2,78^{22}$.

De igual manera la severidad de la enfermedad periodontal estaría asociada a otros factores adicionales tales como el tiempo que el paciente fue diagnosticado con diabetes, o el control metabólico. En la regresión lineal multivariante separada del estudio, se demostró que el número de dientes afectados, se asoció con la edad, el sangrado y la cantidad de biopelícula solo en los sujetos con diabetes ${ }^{22}$.

Por otra parte, un estudio llevado por Lalla y colaboradores en el 2006 comparó a 182 niños y adolescentes diabéticos, con 160 niños y adolescentes no diabéticos. En este estudio el porcentaje de inflamación gingival fue mayor para el grupo de diabéticos $(23,6 \%)$ en comparación con el grupo control $(10,1 \%)$ siendo esta diferencia estadísticamente significativa. La pérdida clínica de inserción (mayor a $2 \mathrm{~mm}$ ), así como el número de dientes afectados fue claramente mayor en el grupo de pacientes diabéticos (promedio:5,8 y 1,8 respectivamente) que el grupo control $(1,5$ y 0,8$)$, siendo esta diferencia estadísticamente significativa ${ }^{23}$.

\section{Sobrepeso}

En el consenso realizado en el Workshop 2017, se considera a la obesidad y al sobrepeso como un factor modificante en la enfermedad periodontal ${ }^{24}$.

Se menciona en la literatura que la asociación de sobrepeso u obesidad y enfermedades periodontales, estaría relacionado a patrones dietéticos deficientes, como baja ingesta de calcio y vitamina $\mathrm{C}$ lo que se traduce en una respuesta inmune deterio$\operatorname{rada}^{24}$.

Un estudio realizado en Estados Unidos en niños con síndrome metabólico, con problemas de sobrepeso y obesidad, demostró que estos presentaron mayores niveles de factor de necrosis tumoral alfa en el líquido crevicular y mayores sitios de sangrado gingival al ser comparado con niños sanos ${ }^{25}$.

Otro estudio llevado a cabo en México en preescolares demostró que los pacientes con tendencia al sobrepeso tenían peor control de biopelícula, o sus niveles de biopelícula visible eran mayores que los pacientes sanos. Además, se encontró una asociación positiva del grupo de biopelícula visible y sobrepeso, inclusive existió una asociación positiva del grupo de placa no visible, sobrepeso y gingivitis ${ }^{14}$.

No obstante en el estudio se encuentra un dato controversial pues en la comparación entre el grupo de presencia de placa visible y peso normal $(0,28 \pm 0,39$, presencia: 19 , $7 \%)$ frente al grupo de presencia de placa visible y riesgo de sobrepeso o sobrepeso $(0,28 \pm 0,36$, presencia: $12,5 \%)$, no se observaron diferencias estadísticamente significativas y ambos grupos mostraron una media alta de gingivitis, lo que podría sugerir que el rol de la cantidad de placa bacteriana es más importante per se, que el de la obesidad como tal' ${ }^{14}$. 


\section{Discusión}

Varios autores ${ }^{1,13,23,24}$ consideran que la cantidad de biopelícula, debido a una deficiente calidad de higiene está asociada a la aparición y severidad de las periodontopatías. No obstante, los porcentajes de esta, pueden variar de acuerdo al país o la región. Como se citó en parágrafos anteriores el porcentaje de pacientes afectados por gingivitis puede variar desde un 34 a 77 por ciento en Latinoamérica ${ }^{1}$. Este valor puede variar un poco en otras regiones y depender del tipo de dentición; según Nobre y cols ${ }^{26}$ la presencia de periodontopatías es mucho menor en la dentición decidua, y mucho menos severa (basado en los códigos de PSR), siendo nuevamente la gingivitis la principal patología en esta edad; por otro lado, la dentición mixtas puede verse afectada por periodontitis, y aunque el porcentaje es bajo (2,5 por ciento), la principal razón a una mayor acumulación de biopelícula, debido al cambio en la oclusión del individuo. Tadjoedin²7, evaluó no solo el tipo de dentición o la prevalencia de enfermedad, sino también su asociación con diferentes grupos etarios. Aunque el principal grupo que presento periodontopatías severas / periodontitis es el grupo que corresponde a adultos y tercera edad, se encontró que en pacientes adolescentes, esta enfermedad puede estar presente, encontrándose una leve correlación entre severidad de la enfermedad y la edad.

Chafe en el 2010 realizó una revisión sistemática con respecto a la asociación de obesidad y enfermedad periodontal; después del análisis de 70 estudios, 41 sugirieron una asociación positiva entre estas dos enfermedades con una razón de riesgo de $1.35^{28}$. Además el estudio concluye que la plausibilidad biológica de este fenómeno se atribuye a una mayor producción de citoquinas pro inflamatorias en el paciente obeso $^{28}$. Scorzetti acota que el tejido adiposo secreta una serie de Interleuquinas y hormonas durante los procesos inflama- torios, los mismos que tendrían caminos similares y fisio-patológicos comunes con la periodontitis ${ }^{29}$; no obstante, estos mecanismos biológicos aún no han sido esclarecidos del todo. Scorzetti también aclara en su estudio que el grupo test de pacientes con un índice de masa corporal aumentado, tuvieron una mayor cantidad de depósitos de biopelícula y con índices de sangrado mayor que el grupo control; con respecto a la pérdida de inserción interproximal no existió diferencia estadísticamente significativa, aunque se encontró una mayor pérdida interproximal en el grupo de pacientes obesos. Finalmente un meta análisis llevado a cabo en el $2017^{30}$ que estudió la asociación de sobrepeso-obesidad y enfermedad periodontal encontró también una asociación positiva con una razón de riesgo igual a 1.46; también se concluye que el odontopediatra debe estar consciente de las posibles alteraciones periodontales en niños con sobrepeso y obesidad ${ }^{30}$.

Con respecto al síndrome de Down también se denota un aumento de la prevalencia y severidad de enfermedad periodontal en personas menores de 35 años con números que varía entre 58 al 96 por ciento. Esto obedecería a factores como una mala higiene como se mencionó con anterioridad, pero también a trastornos de la anatomía dental como raíces cortas o fusionadas, que junto con el aumento de prostaglandinas inflamatorias y alteraciones de grupos celulares de defensa, permitirían el aparecimiento de periodontopatías en paciente con SD de manera más frecuente que en un paciente sin esta condición ${ }^{31}$. Amano ${ }^{32}$ también denota que el aparecimiento temprano enfermedades periodontales que incluyan la pérdida de hueso tendría una edad promedio de $17.1+/-7.1$ años, lo que indicaría que pacientes tendrían algún tipo de enfermedad periodontal incluso antes de la adolescencia específicamente posterior a los 10 años de edad ${ }^{32}$. 


\section{Bibliografía}

1. Botero JE, Rösing CK, Duque $A$, Jaramillo $A$, Contreras A. Periodontal disease in children and adolescents of Latin America. Periodontology 2000. 2015;67(1):34-57.

2. Ibarra MCB. Avaliação do impacto da gengivite na qualidade de vida relacionada à saúde bucal de escolares de 12 anos de Quito-Ecuador: Universidade de São Paulo; 2018.

3. Clerehugh V, Tugnait A. Diagnosis and management of periodontal diseases in children and adolescents. Periodontology 2000. 2001;26(1):14668.

4. Al-Ghutaimel $\mathrm{H}$, Riba H, Al-Kahtani S, Al-Duhaimi S. Common periodontal diseases of children and adolescents. International journal of dentistry. 2014;2014.

5. Orozco Jaramillo R, Peralta Lailson H, Palma Montoya GG, Pérez Rodríguez E, Arróniz Padilla $\mathrm{S}$, Llamosas E. Prevalencia de gingivitis en adolescentes en el municipo de Tlalnepantla. Rev ADM. 2002;59(1):16-21.

6. Orozco Jaramillo R, Peralta Lailson H, Palma Montoya GG, Pérez Rodríguez E, Arróniz Padilla $\mathrm{S}$, Llamosas $\mathrm{H}$. Prevalencia de gingivitis en adolescentes en el municipo de Tlalnepantla. Rev ADM. 2002;59(1):16-21.

7. Biazevic MGH, Rissotto RR, Michel-Crosato E, Mendes LA, Mendes MOA. Relationship between oral health and its impact on quality of life among adolescents. Brazilian oral research. 2008;22(1):36-42.

8. Soto L, Tapia R, Jara G, Rodríguez G, Urbina T, Venegas C. Diagnóstico nacional de salud bucal del adolescente de 12 años y evaluación del grado de cumplimiento de los objetivos sanitarios de salud bucal 2000-2010. Santiago de Chile: Facultad de Odontología, Universidad Mayor. 2007.

9. Salud. MdSPdEOPdl. Estudio epidemiológico de salud bucal en escolares fiscales menores de 15 años en el Ecuador. . Quito, Ecuado; 1995-1996.

10. Lindhe JL NK, T. . Periodontologia Clínica e Implantología Odontológica. . 5TA ed ed2009.

11. Lang NP, Bartold PM. Periodontal health. Journal of periodontology. 2018;89:S9-S16.

12. Van Der Weijden F, Slot DE. Oral hygiene in the prevention of periodontal diseases: the evidence. Periodontology 2000. 2011;55(1):104-23.
13. Juárez-López MLA, Murrieta-Pruneda JF, Teodosio-Procopio E. Prevalencia y factores de riesgo asociados a enfermedad periodontal en preescolares de la Ciudad de México. Gaceta médica de México. 2005;141(3):185-9.

14. Patiño Marín N, Zavala Alonso N, Martínez Castañón G, Alegría Torres J, Medina Solís C, Laredo Naranjo M, et al. Association between dental hygiene, gingivitis and overweight or the risk of overweight in primary teeth of 4 and 5 year old preschoolers in México. International journal of dental hygiene. 2018;16(3):411-8.

15. Akcalı A, Lang NP. Dental calculus: the calcified biofilm and its role in disease development. Periodontology 2000. 2018;76(1):109-15.

16. Caballero A, Ricaurte M, Conrado C. Cálculo dental: una revisión de literatura y presentación de una condición inusual. Acta odontológica venezolana. 2011;49(3).

17. Ferreira R, Michel RC, Greghi SLA, Resende MLRd, Sant'Ana ACP, Damante CA, et al. Prevention and periodontal treatment in Down syndrome patients: a systematic review. PloS one. 2016;11(6):e0158339.

18. Morgan J. Why is periodontal disease more prevalent and more severe in people with Down syndrome? Special Care in Dentistry. 2007;27(5):196-201.

19. Desai SS. Down syndrome: a review of the literature. Oral Surgery, Oral Medicine, Oral Pathology, Oral Radiology, and Endodontology. 1997;84(3):279-85.

20. Lalla E, Cheng B, Lal S, Kaplan S, Softness B, Greenberg E, et al. Diabetes mellitus promotes periodontal destruction in children. Journal of clinical periodontology. 2007;34(4):294-8.

21. Manouchehr-Pour M, Bissada NF. Periodontal disease in juvenile and adult diabetic patients: a review of the literature. Journal of the American Dental Association (1939). 1983;107(5):766-70.

22. Dakovic D, Pavlovic MD. Periodontal disease in children and adolescents with type 1 diabetes in Serbia. Journal of periodontology. 2008;79(6):987-92.

23. Lalla E, Cheng B, Lal S, Tucker S, Greenberg E, Goland R, et al. Periodontal changes in children and adolescents with diabetes: a case-control study. Diabetes care. 2006;29(2):295-9.

24. Chapple IL, Mealey BL, Van Dyke TE, Bartold PM, Dommisch H, Eickholz P, et al. Periodontal health and gingival diseases and conditions on 
an intact and a reduced periodontium: Consensus report of workgroup 1 of the 2017 World Workshop on the Classification of Periodontal and Peri-Implant Diseases and Conditions. Journal of periodontology. 2018;89:S74-S84.

25. Kâ K, Rousseau MC, Lambert M, Tremblay A, Tran SD, Henderson M, et al. Metabolic syndrome and gingival inflammation in Caucasian children with a family history of obesity. Journal of clinical periodontology. 2013;40(11):986-93.

26. Nobre CG, Fernandes-Costa A, de Melo Soares M, Pugliesi DC, de Vasconcelos Gurgel B. Periodontal disease detection in primary and mixed dentitions. European Archives of Paediatric Dentistry. 2016;17(5):407-11.

27. Tadjoedin FM, Fitri AH, Kuswandani SO, Sulijaya $\mathrm{B}$, Soeroso $\mathrm{Y}$. The correlation between age and periodontal diseases. Journal of International Dental and Medical Research. 2017;10(2):327.
28. Chaffee BW, Weston SJ. Association between chronic periodontal disease and obesity: a systematic review and meta-analysis. Journal of periodontology. 2010;81(12):1708-24.

29. Scorzetti L, Marcattili D, Pasini M, Mattei A, Marchetti E, Marzo G. Association between obesity and periodontal disease in children. Eur J Paediatr Dent. 2013;14(3):181-4.

30. Martens L, De Smet S, Yusof M, Rajasekharan S. Association between overweight/obesity and periodontal disease in children and adolescents: a systematic review and meta-analysis. European Archives of Paediatric Dentistry. 2017;18(2):6982.

31. Alrayyes S, Hart TC. Periodontal disease in children. Disease-a-Month. 2011;57(4):184-91.

32. Amano A, Murakami J, Akiyama S, Morisaki I. Etiologic factors of early-onset periodontal disease in Down syndrome. Japanese Dental Science Review. 2008;44(2):118-27.

\section{CITAR ESTE ARTICULO:}

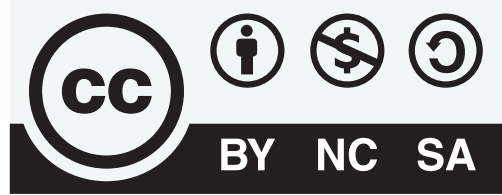

CREATIVE COMMONS RECONOCIMIENTO-NOCOMERCIAL-COMPARTIRIGUAL 4.0.

Franco Mejía, A. J., \& Balseca Ibarra, M. C. (2021). Enfermedad periodontal, prevalencia y factores de riesgo en niños y adolescentes. Revisión de la literatura. RECIMUNDO, 5(3), 359-367. https://doi.org/10.26820/recimundo/5.(3). sep.2021.359-367 